\title{
Substructure Evolution of Ti-6Al-2Zr-1Mo-1V Alloy Isothermally Hot Compressed in $\alpha+\beta$ Two-Phase Region
}

\author{
Chuan WU, He YANG ${ }^{\dagger}$ and Hongwei LI
}

State Key Laboratory of Solidification Processing, School of Materials Science and Engineering, Northwestern Polytechnical University, Xi'an 710072, China

[Manuscript received 23 March 2013, in revised form 11 June 2013]

(C) The Chinese Society for Metals and Springer-Verlag Berlin Heidelberg

\begin{abstract}
Substructure evolution significantly influences the flow behavior of titanium alloys in isothermal hot compression. This paper presents a physical experiment (isothermal hot compression and electron backscatter diffraction, EBSD) and a cellular automaton (CA) method to investigate the substructure evolution of a near$\alpha$ titanium alloy Ti-6Al-2Zr-1Mo-1V (TA15) isothermally compressed in the $\alpha+\beta$ two-phase region. In the CA model, the subgrain growth, the transformation of low angle boundaries (LABs) to high angle boundaries ( $\mathrm{HABs}$ ) and the dislocation density evolution were considered. The dislocation density accumulating around the subgrain boundaries provided a driving force and made the transformation of the LABs to HABs. The CA model was employed to predict the substructure evolution, dislocation density evolution and flow stress. In addition, the effects of strain, strain rate and temperature on the relative frequency of the HABs were analyzed and discussed. To verify the CA model, the predicted results including the relative frequency of the HABs and the flow stress were compared with the experimental values.
\end{abstract}

\section{KEY WORDS: Substructure evolution; Cellular automaton modeling; Dislocation density} evolution; EBSD technique

\section{Introduction}

The Ti-6Al-2Zr-1Mo-1V alloy (TA15) is a type of near- $\alpha$ titanium alloy that possesses a unique combination of properties such as high strength and toughness, low density, good corrosion resistance and excellent high-temperature properties ${ }^{[1]}$. Therefore, the TA15 alloy has been widely used in the aviation and aerospace industry to produce the compressor blades and compressor disks of gas turbine engines, as well as the large front fans of modern jet engines.

In general, thermomechanical processing is the most applicable forming approach for this alloy because of its high yield strength and relatively low elastic modulus. It is well known that the microstructure not only experiences a complex evolution during this process but is also significantly affected by processing parameters such as temperature, strain and strain

† Corresponding author. Prof., Ph.D.; Tel.: +86 298849 5632; E-mail address: yanghe@nwpu.edu.cn (He YANG)

DOI: $10.1007 / \mathrm{s} 40195-013-0161-5$ rate. This indicates that a strong interactive relationship between the microstructural evolution and deformation behavior exists ${ }^{[2]}$. In recent years, much experimental work has been done regarding this subject in terms of microstructural evolution and deformation behaviors.

Fan et al. ${ }^{[3]}$ investigated the effect of deformation inhomogeneity on the microstructural evolution of a large-scale rib-web component of the TA15 alloy under local loading forming. They found that four different microstructures can be obtained during this forming process. They also found that the volume fraction of the primary $\alpha$ phase increases with strain, while the average grain size decreases. Sun et al. ${ }^{[4]}$ investigated the dynamic recrystallization (DRX) and the softening mechanism of a deformed TA15 alloy in the $\alpha+\beta$ two-phase region. Moreover, they developed an internal-state-variable model to simulate the microstructural evolution. Their predicted results, such as the dislocation density and the average recrystallized grain size, agreed well with the experimental values. By the electron backscattered diffraction (EBSD) 
tenichque, Zhu et al. ${ }^{[5]}$ and Huang et al. ${ }^{[6]}$ confirmed that a continuous dynamic recrystallization (CDRX) of titanium alloys such as the TA15 and the Ti-6.5Al3.5Mo-1.5Zr-0.3Si was achieved by the transformation of low angle boundaries (LABs) to high angle boundaries (HABs), during which the flow stress was reduced.

Apart from the aforementioned microstructural evolutions investigated by experimental approach, various meso-scale models, e.g. the Monte Carlo $(\mathrm{MC})^{[7]}$ model and the cellular automaton $(\mathrm{CA})^{[8-11]}$ model, have been widely used to study the microstructural evolution during hot deformation. These models can not only predict the general microstructural properties, but also describe a visible and virtual microstructural evolution in detail. Ding and Guo ${ }^{[12]}$ were the first to couple the CA method with fundamental metallurgical principles to simulate quantitatively and topographically the DRX of the Ti$6 \mathrm{Al}-4 \mathrm{~V}$ alloy during thermo-mechanical processing. The dislocation density evolution, the growth kinetics of recrystallized grains and the flow stress were successfully predicted. Chen et al. ${ }^{[13]}$ coupled the CA method with a topology deformation technique to simulate the microstructural evolution and DRX. Subsequently, Zheng et al. ${ }^{[14]}$ and Lan et al. ${ }^{[15]}$ coupled a crystal plasticity finite element method (CPFEM) with a CA model to study the effect of stored energy of deformation on the recrystallization behavior. However, there have been few studies conducted on the numerical simulation of substructure evolution during isothermal hot compression at the mesoscale, especially, there is little information concerning the effects of processing parameters on the substructure evolution of the TA15 alloy and the explanation of flow softening phenomenon in terms of the substructure evolution.

Therefore, the objective of this study is to investigate the substructure evolution through the CA modeling and EBSD technique. First, a CA model considering the dislocation density evolution, the subgrain growth and the transformation of the LABs to the HABs was developed. Second, the CA model was used to study the effects of strain, strain rate and temperature on the substructure evolution. To verify the current CA model, the predicted results were compared with the experimental data obtained using the EBSD technique. Finally, the effect of substructure evolution on the flow stress and dislocation density was discussed.

\section{Experimental}

\subsection{Experimental material}

The measured chemical composition of used TA 15 alloy (in wt.\%) was $\mathrm{Al} \mathrm{6.8,} \mathrm{Mo} \mathrm{1.7,} \mathrm{V} \mathrm{2.2,} \mathrm{Zr} \mathrm{2.0,} \mathrm{Fe}$ 0.25 , others 0.38 and Ti balanced. The corresponding $\alpha \rightarrow \beta$ transition temperature was $990{ }^{\circ} \mathrm{C}$. The cylin- drical specimens have dimensions of $12 \mathrm{~mm}$ in height and $8 \mathrm{~mm}$ in diameter and were electro-discharged from the received machined material (forged billet).

\subsection{Isothermal hot compression}

The isothermal compression experiments were performed at a temperature range of 900 to $960{ }^{\circ} \mathrm{C}$ and at a strain rate range of 0.01 to $1.0 \mathrm{~s}^{-1}$ on a Gleeble-3800D simulator. Prior to the deformation, each specimen was held at the tested temperature for 10-15 min to ensure a uniform temperature distribution throughout the specimen. The deformation temperature was measured by a thermocouple that was spot-welded to the center of the specimen surface. To reduce the die friction and ensure uniform deformation, two thin pieces of thallium were affixed to the flat dies. All of the compression tests were performed in an argon atmosphere. To preserve the microstructure at high temperatures, the compressed samples were water-quenched immediately after the compression tests. The measured stress strain curves at different deformation conditions are shown in Fig. 1.

\subsection{EBSD measurement}

Following the hot compression experiments, the EBSD technique was used to analyze the subgrain boundaries and local orientation. The deformed specimens were cut along their length axis and mechanical ground using grit papers with different particle sizes ranging from 80 to 2000 meshes. To achieve the surface quality required for the EBSD examinations, the samples were further mechanically polished with the solution of $\mathrm{H}_{2} \mathrm{O}_{2}(30 \mathrm{vol} . \%)$ plus colloidal silica (OP-S, 70 vol.\%). Finally, the prepared samples were examined on a JEOL $6500 \mathrm{~F}$ scanning electron microscope equipped with an EBSD system operating at a voltage of $20-30 \mathrm{~V}$ and a current of $2.2-5.5 \mathrm{nA}$. To analyze the evolution of subgrain boundaries in the alpha phase, each sample was scanned twice. The first scan captured a larger zone containing many primary $\alpha$ grains; the scanning step length was set to $0.5 \mu \mathrm{m}$. The second scan captured a smaller zone to identify the remained $\beta$ with lamellar morphology in a transformed $\beta$ phase; this step length was set to $0.1 \mu \mathrm{m}$. Finally, the subgrain boundary structure, grain morphology and local orientation were analyzed by CHANNEL5 software.

The initial microstructure of the received-material is shown in Fig. 2, where the microstructure consists of a fine, bimodal $\alpha+\beta$ structure of approximately $60 \%$ (volume fraction) equiaxed primary $\alpha$ phase $\left(\alpha_{\mathrm{P}}\right.$, denoted by the white arrow) and $40 \%$ transformed $\beta$ phase $\left(\beta_{\mathrm{T}}\right)$, where the secondary $\alpha$ phase $\left(\alpha_{\mathrm{S}}\right)$ and the retained $\beta$ phase $\left(\beta_{\mathrm{R}}\right)$ are arranged in a lamellar structure with an average grain diameter of approximately $20 \mu \mathrm{m}$. Due to the large difference in grain size between the $\alpha_{\mathrm{P}}$ phase and the $\alpha_{\mathrm{S}}$ phase, the morphol- 

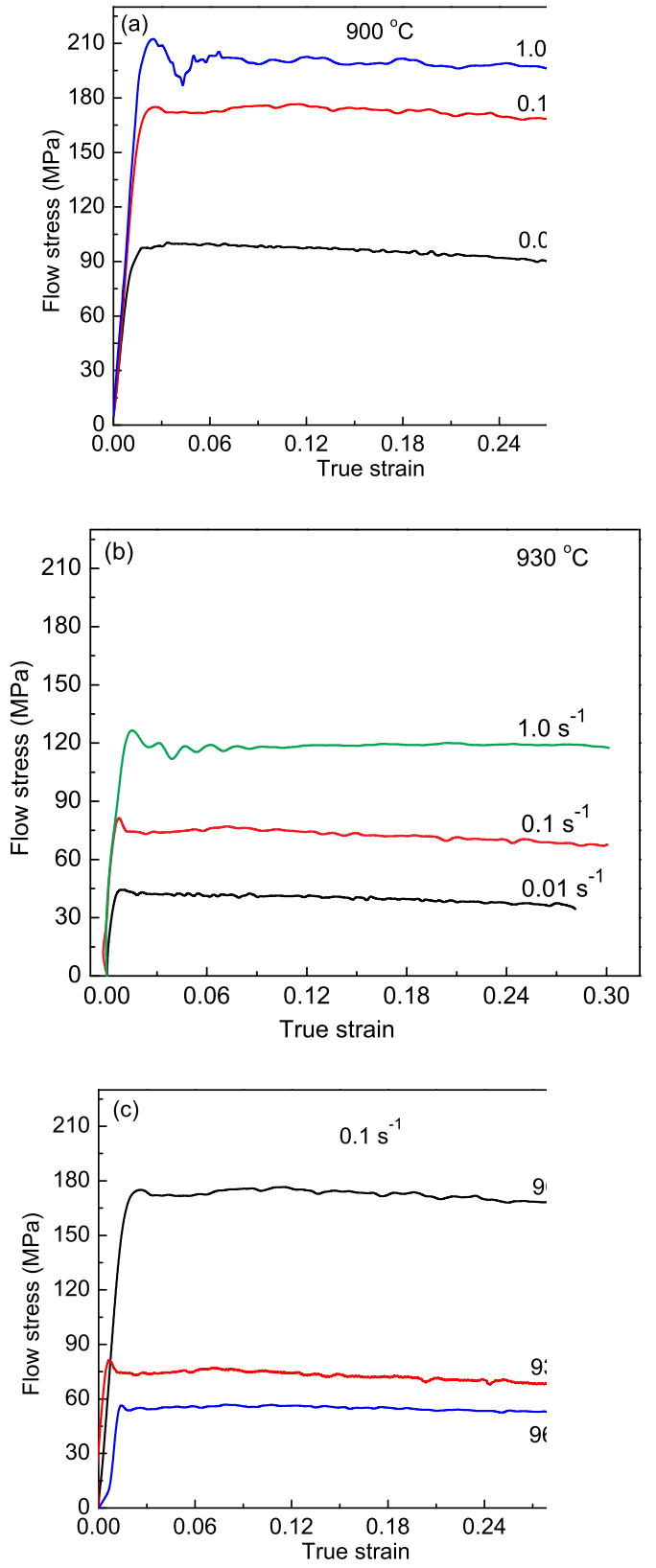

Fig. 1 Flow stress obtained by the isothermal compression at different strain rates and different temperatures: (a) $900{ }^{\circ} \mathrm{C}, 0.01-1.0 \mathrm{~s}^{-1}$; (b) $930{ }^{\circ} \mathrm{C}$, $0.01-1.0 \mathrm{~s}^{-1}$; (c) $900-960{ }^{\circ} \mathrm{C}^{-1}, 0.1 \mathrm{~s}^{-1}$

ogy of the $\alpha_{\mathrm{S}}$ and the $\beta_{\mathrm{R}}$ phase cannot be properly displayed in Fig. 2(a). Therefore, an enlarged image of the microstructure (denoted by a black square) in Fig. 2(a) is shown in Fig. 2(b), where the $\alpha_{\mathrm{S}}$ phase and the $\beta_{\mathrm{R}}$ phase are shown more distinctly. Their average lath thickness is close to $0.2-0.5 \mu \mathrm{m}$. The difference in grain size between the $\alpha_{\mathrm{P}}$ and the $\alpha_{\mathrm{S}}$ (or the $\beta_{R}$ ) can also be displayed in Fig. 3(a), where the $\alpha_{\mathrm{S}}$ and $\beta_{\mathrm{R}}$ cannot be displayed and the grain boundaries between them are blurred. The substructures in the $\alpha_{\mathrm{P}}$ are shown by the red arrow. Fig. 3(b) shows the relative frequency of the HABs and the LABs in the initial microstructure.
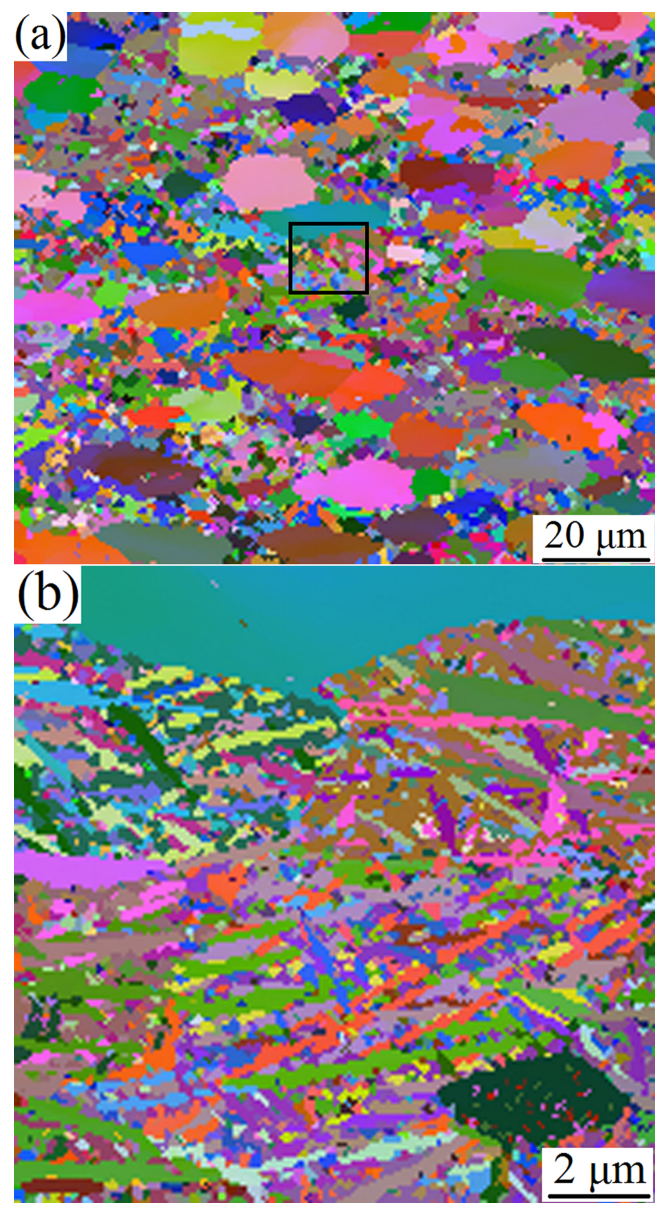

Fig. 2 EBSD image of microstructure in as-received material: (a) microstructure of as-received material; (b) local enlargement of transformed $\beta$ matrix

\section{CA Model for Substructure Evolution}

\subsection{Model of subgrain growth}

It is generally accepted that subgrain growth occurs by two different mechanisms, namely, subgrain boundary migration, and subgrain rotation and coalescence. However, many studies have indicated that the subgrain rotation or coalescence will occur only for subgrains with very small sizes $(\sim 15 \mathrm{~nm})$ and small misorientations and at very high temperatures $\left(>0.9 T_{\mathrm{m}}\right.$, where $T_{\mathrm{m}}$ is the melting point temperature of material) ${ }^{[16]}$. Therefore, in the present study, we are only concerned with the subgrain growth controlled by the migration of subgrain boundaries. It is assumed that the subgrains are spherical, only the subgrain boundaries with the LABs are mobile. During the subgrain growth, the migration rate of subgrain boundaries is expresses as

$$
v=M P
$$

where $v$ is the migration rate, $M$ is the migration mobility of boundaries and $P$ is the driving pressure. 

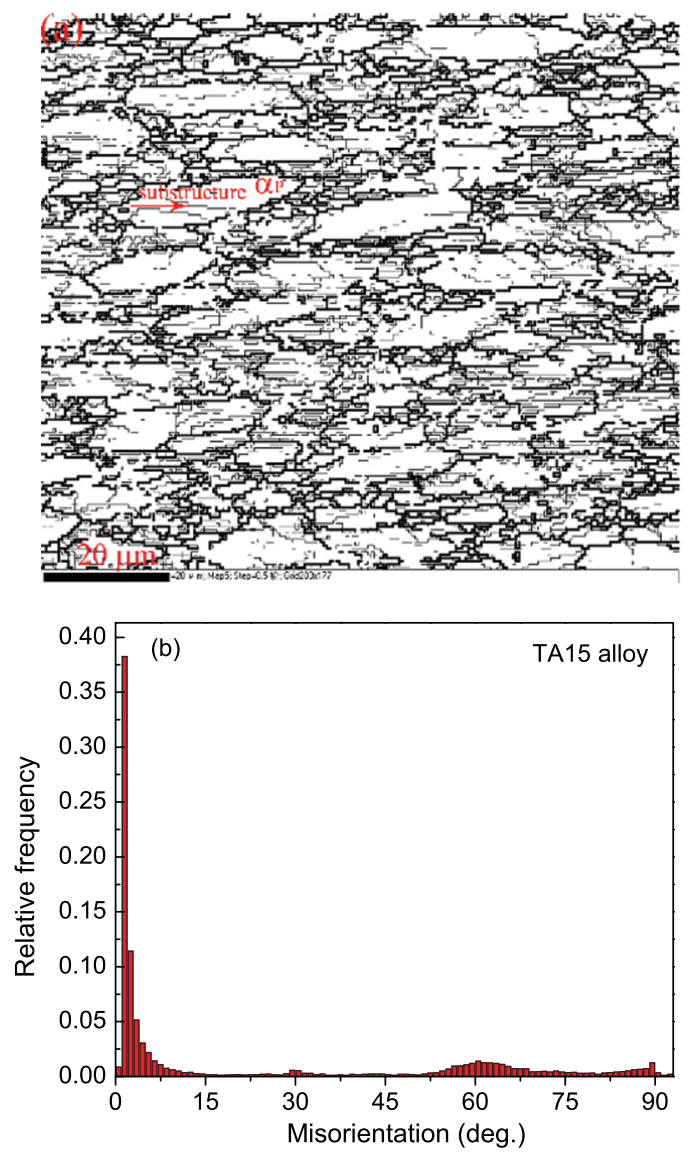

Fig. 3 Grain boundaries microstructure of $\alpha_{\mathrm{P}}, \alpha_{\mathrm{S}}, \beta_{\mathrm{R}}$ subgrain (a) and the relative frequency of HABs measured by EBSD technique (b)

The migration mobility $M$ is expressed as ${ }^{[17]}$

$$
M=\frac{b \delta D_{0}}{k_{\mathrm{B}} T} \exp \left(-\frac{Q_{\mathrm{diffu}}}{R T}\right)
$$

where $b$ is the magnitude of Burgers vector, $\delta$ is the thickness of characteristic boundary, $D_{0}$ is the diffusion coefficient at $0 \mathrm{~K}$ and $Q_{\text {diffu }}$ is the self-diffusion activation energy per mole, $k_{\mathrm{B}}$ is the Boltzmann constant, $R$ is the gas constant and $T$ is the absolute temperature. The driving pressure provided by the driving force is assumed to be distributed evenly within a subgrain and is expressed as

$$
P=\frac{F}{4 \pi r^{2}}
$$

where $F$ is the driving force and $r$ is the subgrain radius. The driving force is deduced by a reduction in the total area of LABs. Therefore, the driving force is given by

$$
F=\frac{\mathrm{d}\left(4 \pi r^{2} \gamma\right)}{\mathrm{d} r}
$$

where $\gamma$ is the boundary energy of subgrains, which is a function of the misorientation of the adjacent subgrains and is given by ${ }^{[18]}$

$$
\gamma=\gamma_{\mathrm{m}} \frac{\theta}{\theta_{\mathrm{m}}}\left(1-\ln \frac{\theta}{\theta_{\mathrm{m}}}\right)
$$

where $\gamma_{\mathrm{m}}$ and $\theta_{\mathrm{m}}\left(\theta_{\mathrm{m}}=15^{\circ}\right)$ are the boundary energy and misorientation of a $\mathrm{HAB}, \theta$ is the misorientation of a subgrain boundary between two neighboring subgrains.

\subsection{Model of dislocation density evolution}

During the deformation process, the evolution of dislocation density is considered as a complex process governed by dislocation multiplication and annihilation. In this study, the Kocks-Mecking equation (K$\mathrm{M}$ model $)^{[19]}$ was employed to describe the dislocation evolution. The dislocation multiplication term is given by

$$
\mathrm{d} \dot{\rho}^{+}=k_{1} \sqrt{\rho} \dot{\varepsilon}
$$

where $k_{1}$ is a constant representing the work hardening effect, $\rho$ is the dislocation density, $\ddot{\varepsilon}$ is the strain rate. The dislocation annihilation term, which is realized either by the glide of dislocations of opposite sign on the same glide plane or by the combination of dislocation glide, climb or cross slip on different glide planes, is given by ${ }^{[19]}$

$$
\mathrm{d} \dot{\rho}^{(1)-}=-k_{2} \rho \dot{\varepsilon}
$$

where $k_{2}$ is a function depending on the strain rate and temperature that represents the softening effect. $k_{2}$ is expressed as [20]

$$
k_{2}=k_{20}\left[\dot{\varepsilon} \exp \left(Q_{\text {act }} / R T\right)\right]^{-1 / \omega}
$$

where $k_{20}$ and $\omega$ are fitting constants, $Q_{\text {act }}$ is the activated energy of deformation. During the migration of the HABs, the volume $\mathrm{d} V$ swept by the mobile boundaries is a product of the boundary area per unit volume and the distance covered during a time increment $\Delta t$. The value of $\mathrm{d} V$ is given by ${ }^{[21]}$

$$
\mathrm{d} V=\frac{4 \pi r_{\mathrm{t}}^{2} v \Delta t}{4 \pi r_{\mathrm{t}}^{3} / 3}
$$

Under the assumption of an equal density of positive and negative dislocations, the dislocation density absorbed by the mobile boundaries is given by

$$
\mathrm{d} \rho=\frac{\rho \mathrm{d} V}{2}
$$

Therefore, the reduction rate of dislocation density due to absorption by mobile boundaries is expressed as:

$$
\mathrm{d} \dot{\rho}^{(2)-}=\frac{3 v \rho}{2 r_{\mathrm{t}}}
$$

And the dislocation density of each cell is calculated as following:

$$
\rho_{i}^{t+\Delta t}=\rho_{i}^{t}+\left(\mathrm{d} \dot{\rho}^{+}+\mathrm{d} \dot{\rho}^{(1)-}+\mathrm{d} \dot{\rho}^{(2)-}\right) \times \Delta t
$$

Here, $\rho_{i}^{t+\Delta t}$ and $\rho_{i}^{t}$ represent the dislocation density of the $i$ th cell at time $t+\Delta t$ and $t$, respectively. The 
macroscopic flow stress during isothermal hot compression is related to the average dislocation density, which is given by ${ }^{[22]}$

$$
\sigma=0.5 \mu b \sqrt{\bar{\rho}}
$$

where $\mu$ is the material shear mould and $\bar{\rho}$ is average dislocation density over all the cells. $\mu(\mathrm{GPa})$ is expressed as ${ }^{[23]}$

$$
\mu=\left(49.02-\frac{5.821}{\exp (181 / T)-1}\right)
$$

\subsection{Model of transformation of LABs to $H A B$ s}

In this study, the LABs are assumed to be mobile while the HABs are immobile. During the substructure evolution, the transformation of LABs to HABs is strongly affected by strain, strain rate and temperature, which will be simulated and discussed in the following sections. Furthermore, the surface fraction of the LABs $\left(f_{\mathrm{LABs}}\right)$ and the HABs $\left(f_{\mathrm{HABs}}\right)$ satisfies the following relation:

$$
f_{\mathrm{LABs}}+f_{\mathrm{HABs}}=1
$$

\subsection{CA modeling framework}

In the CA model, each cell consists of several state variables, including the spatial orientation variable, dislocation density variable, phase state variable and growth distance state variable. The spatial orientation of each cell is set randomly ranging from $0^{\circ}$ to $90^{\circ}$. The cells that belong to the same subgrain are considered to have the same orientation value. The misorientation is equal to the difference in spatial orientation between two neighboring cells on the subgrain boundaries. The boundary with a misorientation larger than $15^{\circ}$ is the $\mathrm{HAB}$, otherwise, it is considered to be a LAB. All the cells have the same initial value of dislocation density $\left(1 \times 10^{10} \mathrm{~m}^{-2}\right.$ in this study). As the deformation continues, the dislocation density of each cell experiences the dislocation multiplication, annihilation and absorption. The phase state variable is used to specify whether a grain belongs to the $\alpha$ phase or the $\beta$ phase. The growth distance state variable determines whether the state variables of a cell are changed or not. If the ratio of growth distance to cell size is larger than unit, the state variables of the cell are changed, furthermore, the reduction of the systemic energy is the largest after the change.

In the CA modeling, the microstructural evolution is realized by updating the state variables of each cell based on a switching rule. The switching rule is defined as a function of a previous state of a cell and its neighboring ones, which is mainly formulated based on the models of subgrain growth and transformation of the LABs to the HABs. A complete procedure for the CA modeling of the substructure evolution is summarized as follows:

(1) The initial microstructure for the substructure evolution is generated by using a modified CA model $^{[13]}$

(2) The state variables, including the spatial orientation, dislocation density, phase state and growth distance, are assigned to each cell.

(3) In each CA simulation step (CAS), all of the cells on the subgrain boundaries are selected firstly. Then, the migration mobility, driving pressure and migration rate of the each cell are calculated according to Eqs. (2), (4) and (3), respectively. The growth distance is calculated according to the formula of $l_{i, j}^{t}=\int_{0}^{t} v \mathrm{~d} t$, here the term $l_{i, j}^{t}$ represents the growth distance of the $i$ th cell toward its $j$ th neighboring cell over time $t$. Next, the ratio of $l_{i, j}^{t}$ to the cell size is calculated. If the value of the ratio is less than unit, the state variables of the cell can not be updated. Otherwise, a random number ran_num $(0<$ ran_num $<1)$ is produced by computer program. The random is compared with a transformation probability $\left(P_{\mathrm{t}}\right)$ of the LABs to the HABs. Since the substructure evolution is not only influenced by the reduction of interface energy, but also influenced by the dislocations accumulating around the subgrain boundaries. The dislocation density evolution is sensitive to strain, strain rate and temperature. Thus the $P_{\mathrm{t}}$ is expressed as a function of strain, strain rate and temperature. If the value of ran_num is larger than $P_{\mathrm{t}}$, the state variables of the cell are changed into those of one of its neighboring cell. After the transition, the misorientation between the cell and its neighboring cells is calculated. In the $\mathrm{CA}$ modeling, the relative frequency of the HABs is equal to a ratio of the number of cells representing the HABs to the total number of cells on subgrain boundaries.

The evolution of dislocation density of each cell consists of dislocation multiplication, annihilation and absorption. The dislocation multiplication and annihilation follows the K-M equation. A part of dislocations within subgrains are absorbed by the mobile subgrain boundaries. The dislocation annihilation and absorption can reduce the dislocation density and result in the flow softening. The simulation procedure for the current CA modeling of substructure evolution is illustrated in Fig. 4.

\section{Parameter Determination}

In this study, the substructure evolution of the TA15 alloy during isothermal hot compression in the $\alpha+\beta$ two-phase region was modeled by the CA method. Substructure evolution takes place mainly in the $\alpha_{\mathrm{P}}$ phase. Therefore, only the substructure evolution in the $\alpha_{\mathrm{P}}$ was considered in this model. The experimental stress strain curves were taken from the hot compression experiments to fit the material constants in the CA model. 
Table 1 Material constants of the TA15 alloy used in models

\begin{tabular}{ccccc}
\hline Material & $b\left(\mathrm{~m}^{-1}\right)$ & $\delta D_{0}\left(\mathrm{~m}^{3} / \mathrm{s}\right)$ & $Q_{\text {diffu }}(\mathrm{kJ} / \mathrm{mol})$ & $Q_{\text {act }}(\mathrm{kJ} / \mathrm{mol})$ \\
\hline$\alpha$-Ti & $2.95 \times 10^{-10}$ & $3.6 \times 10^{-16}$ & $97 \times 10^{3}$ & $200^{20]}$ \\
$\beta$-Ti & $2.86 \times 10^{-10}$ & $5.4 \times 10^{-17}$ & $153 \times 10^{3}$ & \\
\hline
\end{tabular}

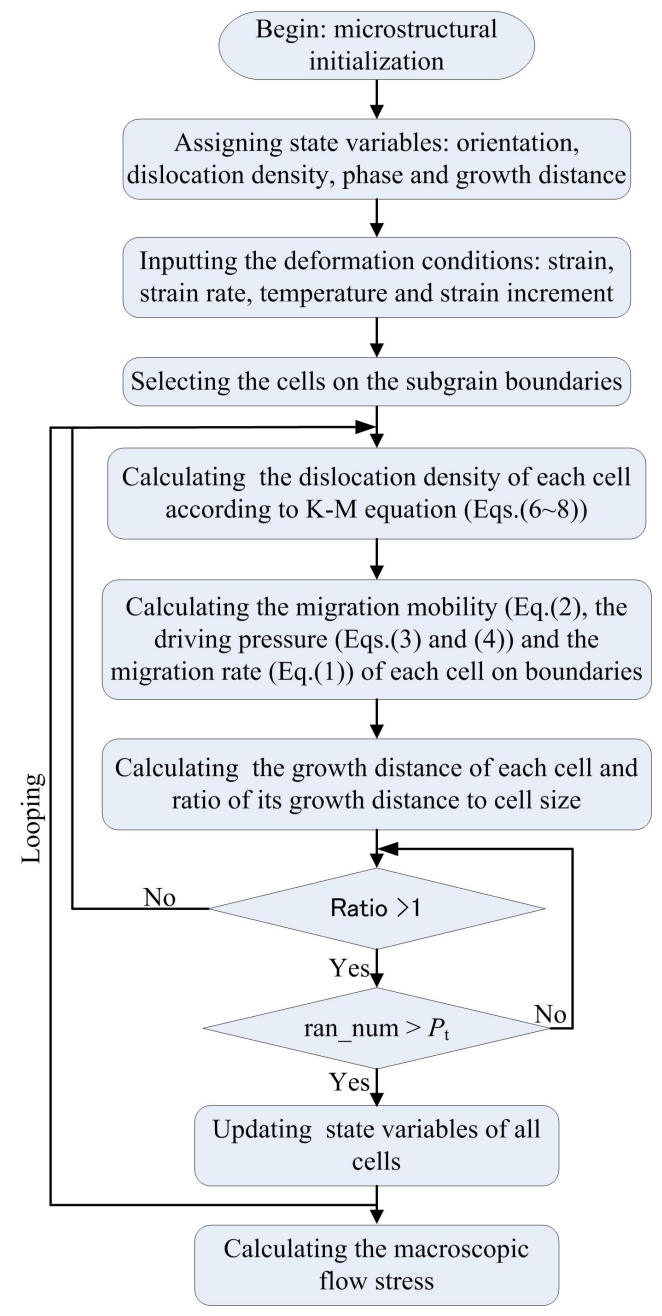

Fig. 4 Chart of CA modeling of substructure evolution

There are several parameters that must be determined in the model. Some of these are physically meaningful and independent of strain, strain rate or temperature, such as the Burgers vector $b$ ( $\alpha$ or $\beta$ phase) in Eq. (2), grain boundary energy of the high angle $\gamma_{\mathrm{m}}$ and high angle $\theta_{\mathrm{m}}$ in Eq. (5), self-diffusion activated energy $Q_{\text {diff }}$ in Eq. (2), thickness of characteristic boundary $\delta$, diffusion coefficient $D_{0}$ at $0 \mathrm{~K}$ in Eq. (2). These values can be directly obtained and are listed in Table 1.

Other constants that must be determined by fitting the experimental data include the constant $k_{1}$ in Eq. (6), the constants $k_{20}$ and exponent $\omega$ in Eq. (8). Although the deformation behavior of the $\alpha$ phase is generally different from that of the $\beta$ phase, however, for simplicity, it is assumes that both the $\alpha$ and $\beta$ phases have the same values for the $k_{1}, k_{20}$ and exponent $\omega$. Constant $k_{1}$ in Eq. (6) represents the increasing rate of the dislocation density due to dislo- cation multiplication. Constant $k_{20}$ in Eq. (8) represents the dislocation annihilation induced by dislocation glide in the same system or dislocation climb or cross slip between different systems ${ }^{[19]}$. In general, the dislocation generation can be gradually balanced by the dislocation annihilation after peak strain, which results in a steady stage. During this stage, the flow stress keeps no change with strain and is assumed to be equal to the peak stress. According to Eq. (13), the order of magnitude of dislocation density in the matrix during the steady state may be estimated. Hence, the ratio of $k_{1}$ to $k_{20}$ can be determined from the equivalence of Eqs. (6) and (7). After this step, the specific values of $k_{1}$ and $k_{20}$ are estimated using an optimization approach such as the genetic algorithm (GA) -based objective optimization technique ${ }^{[24,25]}$. The GA algorithm as a stochastic search method based on evolution and genetics is used to search the most effective solution. For the GA optimization method, the objective function is needed to be defined. In this study, the objective function was defined as

$$
f(x)=\frac{1}{n} \sum_{i}^{n}\left(\xi \mu b \sqrt{\bar{\rho}_{i}}-\sigma_{i}\right)^{2}
$$

where $\bar{\rho}_{i}$ represents the average dislocation density at a specific deformation condition and $\xi b \mu \sqrt{\bar{\rho}_{i}}$ is the corresponding predictive flow stress and $\xi$ is a constant $(\xi=0.5) . n$ is the total number of all deformation conditions. $\sigma_{i}$ is the corresponding experimental value. $\bar{\rho}_{i}$ can be rewritten as

$$
\rho_{t+\Delta t}=\rho_{t}+\left(k_{1} \sqrt{\rho_{t}}-k_{20} Z^{-1 / \omega} \rho_{t}\right) \Delta t \dot{\varepsilon}
$$

where $\rho_{t+\Delta t}$ and $\rho_{t}$ are the dislocation density at time $t+\Delta t$ and $t$, respectively. $\mathrm{Z}$ parameter represents $\dot{\varepsilon} \exp \left(Q_{\text {act }} / R T\right)$. The value of $\omega$ can be directly obtained from Fan and Yang ${ }^{[20]}$. Finally, the specific values of these parameters are $\omega=0.167^{[20]}$, $k_{1}=8 \times 10^{9} \mathrm{~m}^{-1}$, and $k_{20}=1500$.

\section{Simulated Results and Discussion}

\subsection{Initial microstructure}

In this study, the CA method was employed to simulate the substructure evolution of the TA15 alloy during hot compression. The CA space is divided into a $500 \times 500$ square lattices (cells). The size of each cell represents $0.39 \mu \mathrm{m}$ of the real dimension of the material. Accordingly, the simulated area corresponds to $195 \mu \mathrm{m} \times 195 \mu \mathrm{m}$ in a real sample. The initial dislocation density is set to $1.0 \times 10^{10} \mathrm{~m}^{-2[26]}$. The cell is selected as a regular square with a periodic boundary 


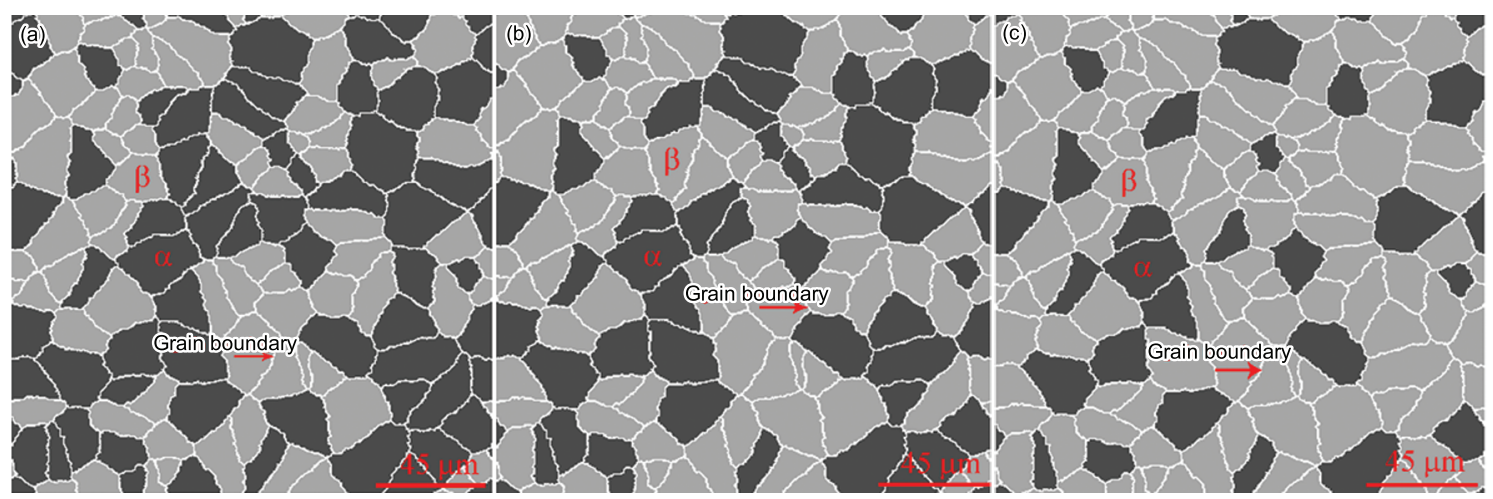

Fig. 5 Initial microstructure of substructure evolution simulated by the $\mathrm{CA}$ modeling: (a) $900{ }^{\circ} \mathrm{C}$, (b) $930{ }^{\circ} \mathrm{C}$, (c) $960{ }^{\circ} \mathrm{C}$

condition and the Moore's neighboring rule. The initial microstructure for the substructure evolution is generated by a modified CA model proposed by Chen et $a .^{[13]}$, where the effects of the thermodynamic driving mechanism, curvature-driven mechanism and lowest energy principle on the microstructural evolution were considered.

Fig. 5(a), Fig. 5(b) and Fig. 5(c) show the simulated microstructures of the TA15 alloy at temperatures of 900,930 and $960{ }^{\circ} \mathrm{C}$, respectively. It can be seen that the morphology is equiaxed. The $\alpha_{\mathrm{P}}$ grains are denoted as the 'black color', while the $\beta_{\mathrm{T}}$ grains (including $\mathrm{S}$ and $\mathrm{R}$ ) are denoted as 'gray color'. The grain boundary is denoted by the red arrow. Clearly, the volume fraction of the $\alpha\left(f_{\mathrm{v}}\right)$ decreases with the increasing temperature. At $900{ }^{\circ} \mathrm{C}$, its volume fraction is up to $56 \%$. When temperature increases to 930 and $960{ }^{\circ} \mathrm{C}$, the values of $f_{\mathrm{v}}$ decrease to $40 \%$ and $25 \%$, respectively, which are close to the experimental values. However, the average grain size nearly remains the same value at these temperatures, which is close to the initial value of $20 \mu \mathrm{m}$ (in diameter). Due to the effect of the $\beta$ retard on the $\alpha$ growth, the coarsening of the $\alpha$ grains is not apparent. Thus the average grain size at temperatures of 900,930 and $960{ }^{\circ} \mathrm{C}$ has the same value.

\subsection{Substructure evolution}

To study the effect of temperature on the substructure evolution, the simulated substructures in the $\alpha_{\mathrm{P}}$ at $0.1 \mathrm{~s}^{-1}$, height reduction of $50 \%$ and temperatures of 900,930 and $960{ }^{\circ} \mathrm{C}$ are shown in Fig. 6(a), Fig. 6(b) and Fig. 6(c), respectively. In these figures, the red lines denote the subgrain boundaries with the HABs while the black lines denote the grain boundaries of the $\alpha$ and $\beta$ phases or the $\alpha-\beta$ phase interface. The intensity of the red lines decreases with temperature, while the intensity of the black lines increases. This may be caused by several factors. First, the volume fraction of the $\alpha_{\mathrm{P}}$ decreases with temperature so that the intensity of the red lines decreases. Second, the volume fraction of subgrain boundaries with the

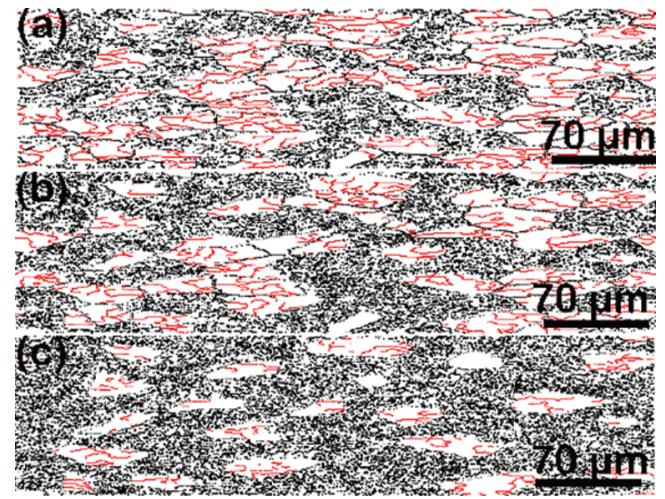

Fig. 6 Effect of temperature on substructure evolution simulated by the CA model at $0.1 \mathrm{~s}^{-1}, 50 \%$ height reduction and temperatures of $900{ }^{\circ} \mathrm{C}$ (a), $930{ }^{\circ} \mathrm{C}$ (b) and $960{ }^{\circ} \mathrm{C}(\mathrm{c})$

LABs transformed into the HABs decreases with temperature so that the intensity of red lines decreases. However, the volume fraction of the $\alpha_{\mathrm{S}}$ (with lamellar structure precipitated in the $\beta_{\mathrm{T}}$ phase) increases with temperature. Thus, the intensity of the black lines $(\alpha-\beta$ phase interface) increases.

Fig. 7(a) to Fig. 7(c) show the predicted relative frequencies of the HABs at the same condition as in Fig. 6. In Fig. 7 (a) $\left(900{ }^{\circ} \mathrm{C}\right)$, a large proportion of the HABs are produced, largely more than that of the LABs, and the relative frequency of the HABs is close to the experimental value of $78.5 \%$ (red-curve). However, the predicted peak values of the LABs are not apparent as those in experiment. When temperature increases to $930{ }^{\circ} \mathrm{C}$, as shown in Fig. 7(b), the predicted relative frequency of the HABs decreases to $54.5 \%$. There is an obvious peak value of the LABs concentrating on a range of $1^{\circ}$ to $5^{\circ}$ in experiments, while the peak value cannot be observed in the $\mathrm{CA}$ modeling and the prediced HABs are distributed evenly. At $960{ }^{\circ} \mathrm{C}$, as shown in Fig. 7(c), the predicted relative frequency of the HABs reduces to $27.7 \%$. This value is very close to the experimental value of $28 \%$. However, the predicted distribution shapes of the relative frequencies of the HABs and LABs are 

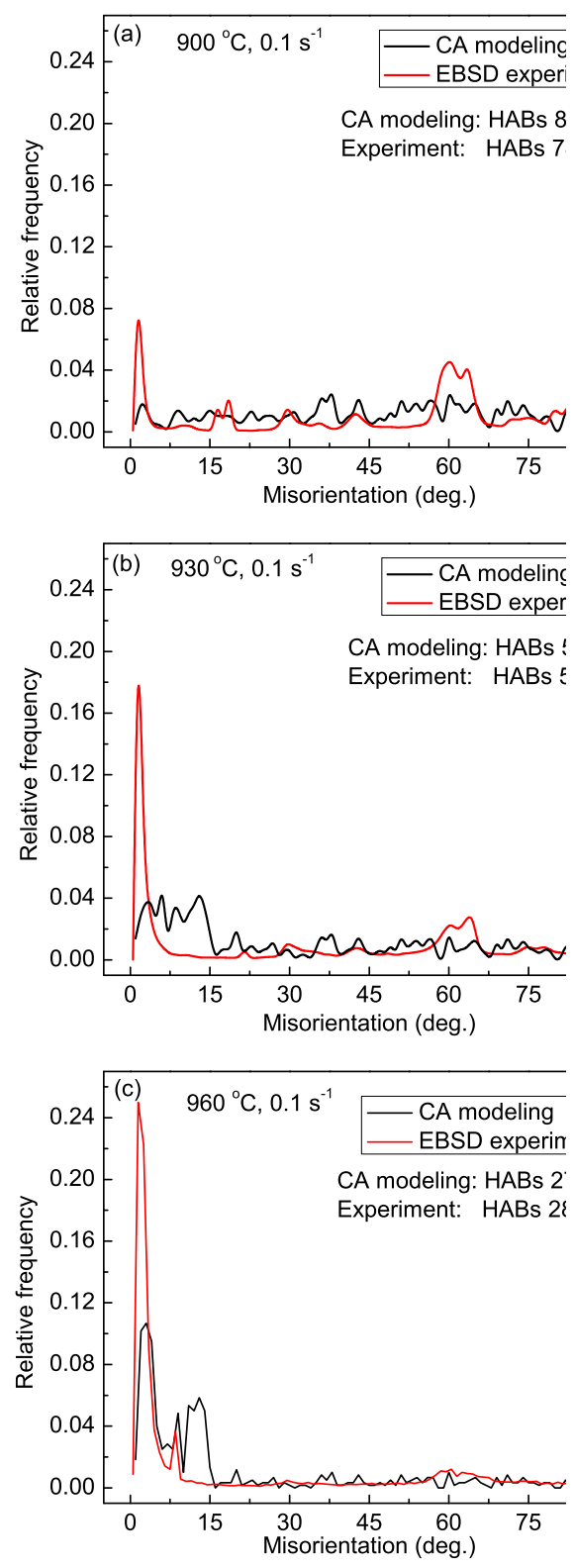

Fig. 7 Comparison of the predictive relative frequency of HABs (LABs) with the experiment at $0.1 \mathrm{~s}^{-1}$, $50 \%$ height reduction and temperatures of $900{ }^{\circ} \mathrm{C}$ (a), $930{ }^{\circ} \mathrm{C}(\mathrm{b})$ and $960{ }^{\circ} \mathrm{C}(\mathrm{c})$

different from those in experiment. Especially for the distribution of the relative frequency of the LABs, two obvious peak values of $1^{\circ}$ to $5^{\circ}$ and $10^{\circ}$ to $15^{\circ}$ the appear in the simulated curve, but there is only one peak value concentrating on $1^{\circ}$ to $5^{\circ}$ in the experimental curve.

During a deformation process, a large number of dislocations are produced inside the deformed grains and become heavily tangled. The dislocations with opposite signs react each other by glide on the same plane or by climb or cross slip between the different slip planes. These types of dislocation reactions lead to the formation of subgrains from regular dislocation cells. With further deformation, a fraction of subgrains with the LABs gradually transform into

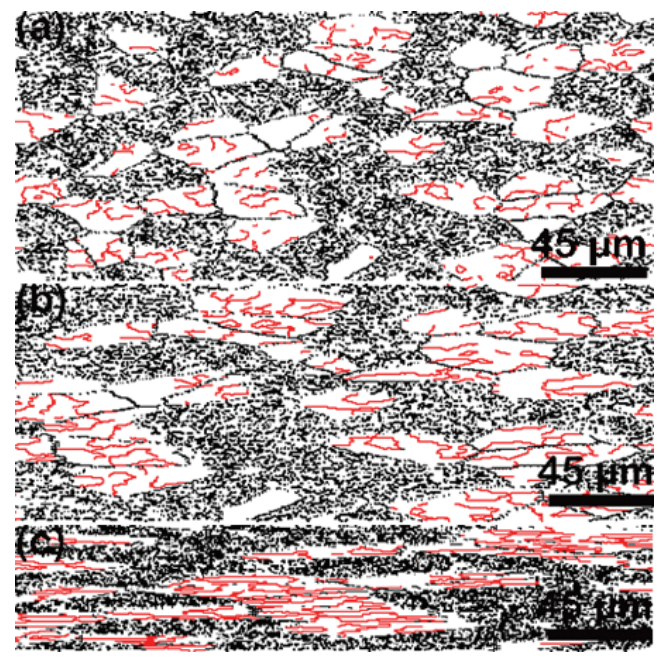

Fig. 8 Effect of strain on substructure evolution simulated by the $\mathrm{CA}$ model at $0.1 \mathrm{~s}^{-1}, 930{ }^{\circ} \mathrm{C}$ and height reductions of $30 \%$ (a), $50 \%$ (b) and $70 \%$ (c)

the HABs. Obviously, the rate of dislocation climb or cross slip increases with increasing temperature, which largely reduces the driving force. This results in the relative frequency of the LABs (or the HABs) at higher temperature larger (smaller) than that at lower temperature.

To investigate the effect of strain on the substructure evolution in the $\alpha_{\mathrm{P}}$ phase, the substructures modeled by the $\mathrm{CA}$ method at $930{ }^{\circ} \mathrm{C}$ and $0.1 \mathrm{~s}^{-1}$ and height reductions of $30 \%, 50 \%$ and $70 \%$ are shown in Fig. 8(a), Fig. 8(b) and Fig. 8(c), respectively. It can be seen that the density of the black lines increases with strain. The aspect ratio of the $\alpha_{\mathrm{P}}$ and the volume fraction of the $\alpha_{\mathrm{S}}$ increase with strain. During the deformation process, there is a competition between the formation of new subgrains with the LABs and their consumption by the transformation of the LABs into the HABs. A large number of subgrains are created from the dislocation cells due to dislocation reaction and arrangement. At the same time, more dislocations produced by the plastic deformation accumulate around the subgrain boundaries, increasing the driving force and promoting a more rapid transformation of the LABs into the HABs. This process reduces the relative frequency of the LABs. Therefore, the density of red lines or the relative frequency of the HABs increases with strain.

Fig. 9(a), Fig. 9(b) and Fig. 9(c) show the relative frequency of the $\mathrm{HABs}$ (or the LABs) at $930{ }^{\circ} \mathrm{C}$, $0.1 \mathrm{~s}^{-1}$ and height reductions of $30 \%, 50 \%$ and $70 \%$, respectively. From these figures, it can be seen that the predicted relative frequency of the HABs or LABs agrees better with the experimental value. As shown in Fig. 9(a), the relative frequency of the HABs at a reduction of $30 \%$ is $47.9 \%$, while this value increases to $59.3 \%$ at a reduction of $50 \%$. As deformation continues, a large number of dislocations produced by plastic deformation gradually accumulate around the 

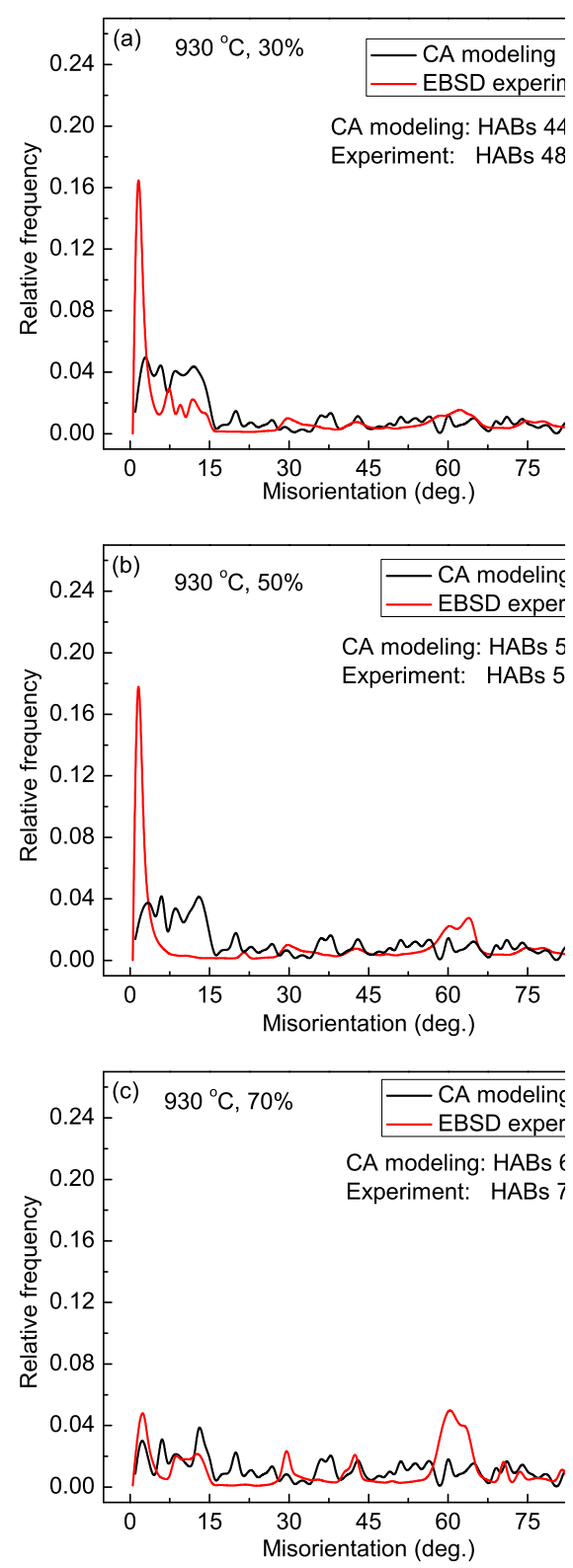

Fig. 9 Comparison of the predictive relative frequency of the HABs with the experiment at $930{ }^{\circ} \mathrm{C}, 0.1 \mathrm{~s}^{-1}$ and height reductions of $30 \%$ (a), $50 \%$ (b) and $70 \%$ (c)

subgrain boundaries, which promotes the transformation of the LABs to the HABs. This process results in the decrease of the LABs. When the reduction increases to $70 \%$ (as shown in Fig. 9(c)), the fraction of the HABs increases to $68.4 \%$ and the misorientation distribution exhibits new peak values of 35,40 , 60 and 90 degrees. Moreover, the relative frequency of the LABs is smaller than that at reduction of $50 \%$ (Fig. 9(b)).

Fig. 10(a) to Fig. 10(c) show the simulated substructure evolution at $930{ }^{\circ} \mathrm{C}, 50 \%$ height reduction and strain rates of 0.01 to $1.0 \mathrm{~s}^{-1}$. From these figures, it can be observed that the volume fraction of the $\alpha_{\mathrm{P}}$ and its morphology are insensitive to the strain rate. However, the distribution of subgrain bound-

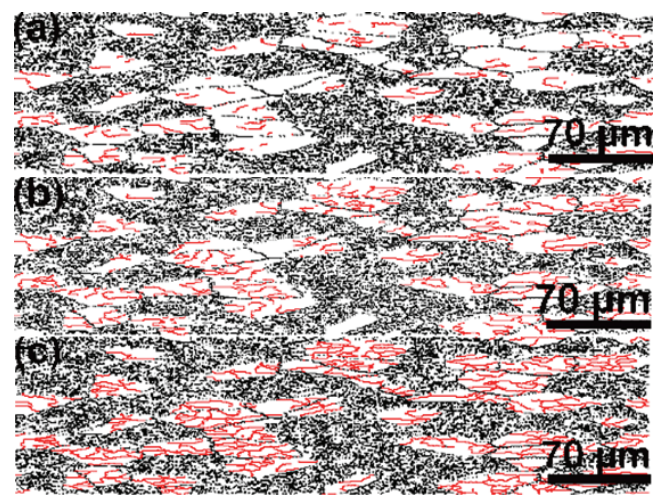

Fig. 10 Effect of strain rate on substructure evolution simulated by the CA model at $930{ }^{\circ} \mathrm{C}, 50 \%$ height reduction and strain rates of $0.01 \mathrm{~s}^{-1}$ (a), $0.1 \mathrm{~s}^{-1}$ (b) and $1.0 \mathrm{~s}^{-1}(\mathrm{c})$

aries and the $\alpha_{\mathrm{S}}$ or $\beta_{\mathrm{R}}$ are significantly influenced by the strain rate. At a lower strain rate of $0.01 \mathrm{~s}^{-1}$ (see Fig. 10(a)), the density of the red (representing the HABs) and black (representing the interface of $\alpha_{\mathrm{S}}-\beta_{\mathrm{R}}$ ) lines is relatively lower compared to that at higher strain rates. The relative frequency of the HABs increases gradually with the increase of strain rate. This is because there is no enough time for the dislocations to take place climb or cross slip so that more and more dislocations begin to accumulate around the subgrain boundaries. These accumulating dislocations can provide the extra energy to drive the transformation of the LABs into the HABs. The density of the red lines increases largely as the strain rate increases to $1.0 \mathrm{~s}^{-1}$. Moreover, the intensity of the black lines (the grain boundaries of $\alpha, \beta$ or $\alpha-\beta$ ) increases as well. As the strain rate increases, there is no enough time for the lamellar $\alpha_{\mathrm{S}}$ or $\beta_{\mathrm{R}}$ to take place globularization, thus the intensity of their interfaces increases.

Fig. 11(a) to Fig. 11(c) show the comparisons of predicted relative frequency of the HABs (or the LABs) with the experimental values at $930{ }^{\circ} \mathrm{C}, 50 \%$ height reduction and strain rates of $0.01,0.1$ and $1.0 \mathrm{~s}^{-1}$, respectively. It can be seen that the relative frequency of the HABs (or the LABs) is sensitive to the strain rate. The relative frequency of the HABs increases with the strain rate. At strain rate of $0.01 \mathrm{~s}^{-1}$ (see Fig. 11(a)), the predicted relative frequency of the HABs is less than $30 \%$, which agrees well with the experimental result. When strain rate increases to $0.1 \mathrm{~s}^{-1}$ ( see Fig. 11(b)) and $1.0 \mathrm{~s}^{-1}$ ( see Fig. 11(c)), the predicted relative frequency of the HABs increases to $54.5 \%$ and $92.3 \%$, respectively, which is apparently larger than that at strain rate of $0.01 \mathrm{~s}^{-1}$. This evolution law may be explained as follows. At a lower strain rate of $0.01 \mathrm{~s}^{-1}$, there is enough time for dislocation glide, climb or cross slip. This can largely reduce the driving force for the transformation of the LABs to the HABs. Thus the relative frequency of the LABs is higher but lower for 

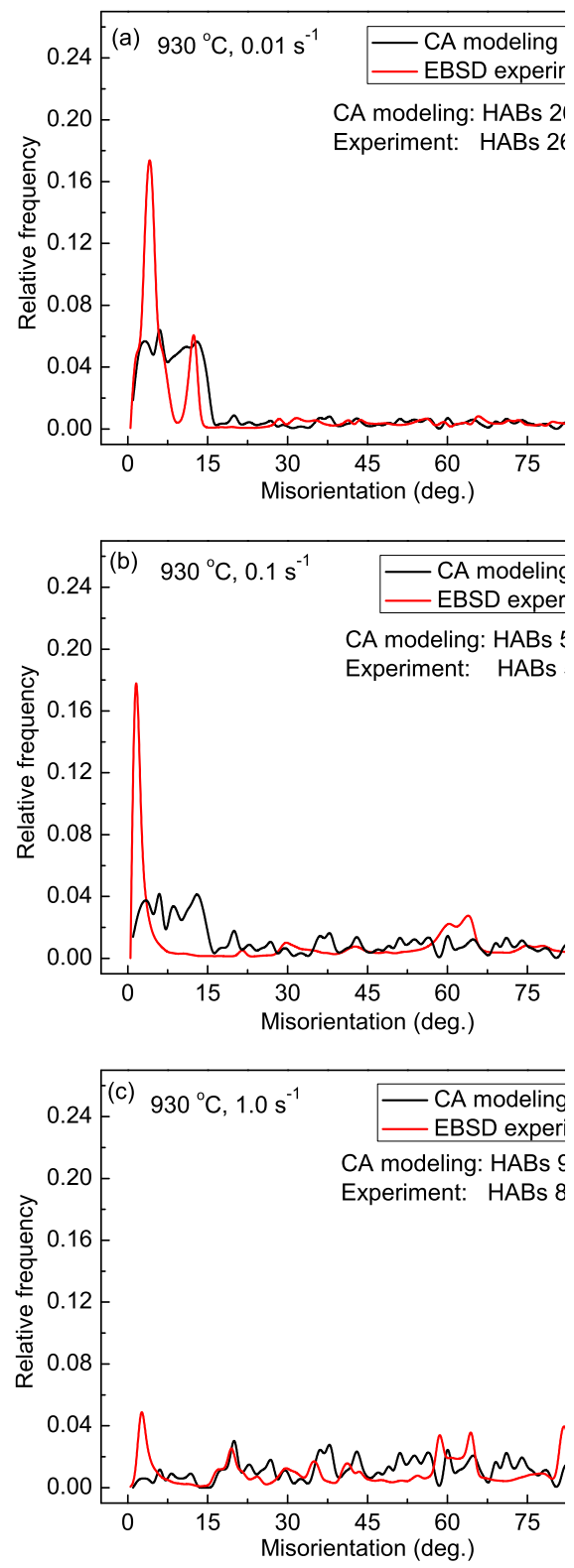

Fig. 11 Comparison of the predictive relative frequency of the HABs with the experimental at $930{ }^{\circ} \mathrm{C}, 50 \%$ height reduction and strain rates of $0.01 \mathrm{~s}^{-1}$ (a), $0.1 \mathrm{~s}^{-1}$ (b) and $1.0 \mathrm{~s}^{-1}$ (c)

the HABs. When the strain rate increases to a higher value of $0.1 \mathrm{~s}^{-1}$ or $1.0 \mathrm{~s}^{-1}$, there is no time for the dislocations to take place glide and climb, hence much more immobile dislocations accumulating around the subgrain boundaries supply the driving force. Therefore, a large fraction of the HABs are formed by the continuous transformation of the LABs to the HABs.

\subsection{Dislocation density evolution}

The evolution of average dislocation density during the substructure evolution is shown in Fig. 12(a), Fig. 12(b) and Fig. 12(c). From these figures, it can be seen that the effects of temperature and strain rate on dislocation density evolution are significant. At
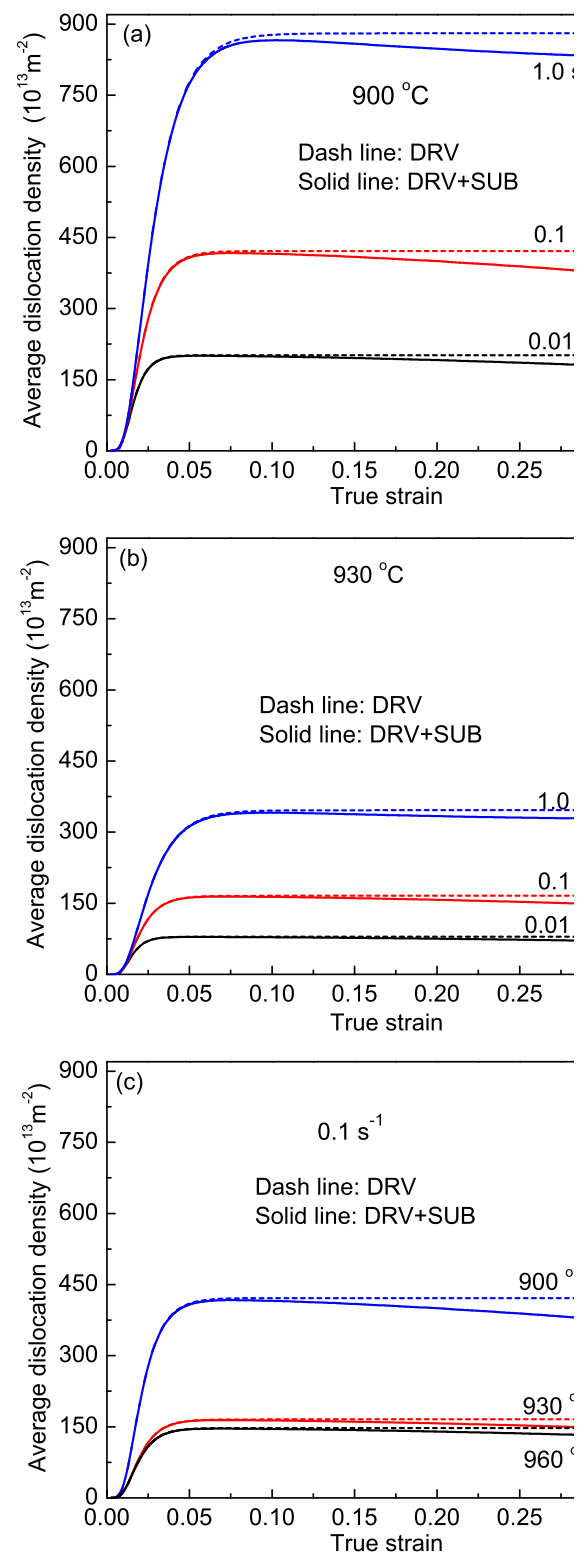

Fig. 12 Dislocation density evolution predicted by the $\mathrm{CA}$ model at different deformation conditions: (a) $900{ }^{\circ} \mathrm{C}$, (b) $930{ }^{\circ} \mathrm{C}$, (c) $0.1 \mathrm{~s}^{-1}$

$900{ }^{\circ} \mathrm{C}$ (see Fig. 12(a)), the saturated value of average dislocation density increases from $150 \times 10^{13}$ to $900 \times 10^{13} \mathrm{~m}^{-2}$ as the strain rate increases from 0.01 to $1.0 \mathrm{~s}^{-1}$. This is because there is no enough time for the dislocations to glide, climb or cross slip as the strain rate increases, thus more and more dislocations accumulate, which results in an increase of dislocation density. Similarly, at $930{ }^{\circ} \mathrm{C}$, the saturated value of average dislocation density increases from $50 \times 10^{13}$ to $300 \times 10^{13} \mathrm{~m}^{-2}$, as shown in Fig. 12(b). At the same strain rate, the average dislocation density decreases with the increasing temperature. This is because the higher the temperature is, the easier the climb or cross slip of dislocations takes place, which reduces the dislocation density.

Fig. 12(a) to Fig. 12(c) also display the effect of 
substructure evolution on dislocation density. From these figures, it is clear that the substructure evolution can reduce the dislocation density. The dash lines represent the average dislocation density, only considering the dynamic recovery (denoted by DRV). In this case, the dislocation density increases rapidly with strain during the initial stages, then it reaches a steady stage in which the dislocation density remains unchangeable with strain. If the substructure evolution is considered in the CA model, the dislocation density decreases with strain after the peak strain. This is represented in these figures by the solid lines (denoted by DRV+SUB). It can be seen that the dislocation density decreases with strain and that a softening phenomenon is obvious. During a deformation process, the dislocations within the subgrains or subgrain boundaries react each other with those of opposite signs by dislocation movements. At the same time, the dislocations accumulating around the subgrain boundaries drive the transformation of the LABs to the HABs. The mobile subgrain boundaries sweep the deformed areas with relatively higher dislocation density. These areas are gradually depleted and the dislocations in these areas are absorbed by the mobile boundaries. During the subgrain evolution, not only the dislocation reaction but the dislocation absorption takes place. Therefore the dislocation density reduces and a softening phenomenon appears.

From these figures, it is also seen that the reduction extent of dislocation density decreases with the increasing temperature or decreasing strain rate. The rate of dislocation glide, climb or cross slip increases with temperature, which results in a reduction of dislocation density accumulating around the subgrain boundaries, the dislocations absorbed by the mobile subgrain boundaries are less. Similarly, the dislocations absorbed by the mobile boundaries decrease with the decreasing strain rate due to the fact that there is enough time for the dislocations to take place dislocation reactions.

\subsection{Predicted flow stress}

The flow stress of the TA15 alloy was predicted at given temperatures $\left(900\right.$ to $930{ }^{\circ} \mathrm{C}$ ) and strain rates $\left(0.01\right.$ to $\left.1.0 \mathrm{~s}^{-1}\right)$. The predicted flow stress is compared with the experimental data, as shown in Fig. 13. It can be seen that the comparisons agree good. However, during the initial deformation stages, the predicted flow stress deviates slightly from the experimental value. In the $\alpha+\beta$ two-phase field, the flow stress significantly increases with the decreasing temperature or increasing strain rate, as shown in these figures. For example, the predicted peak stress increases approximately from 40 to $200 \mathrm{MPa}$ with temperature decreasing from 960 to $900{ }^{\circ} \mathrm{C}$ at a constant strain rate of $0.1 \mathrm{~s}^{-1}$. Meanwhile, at a given temperature, the predicted peak stress increases with the increasing strain rate. These results indicate that the
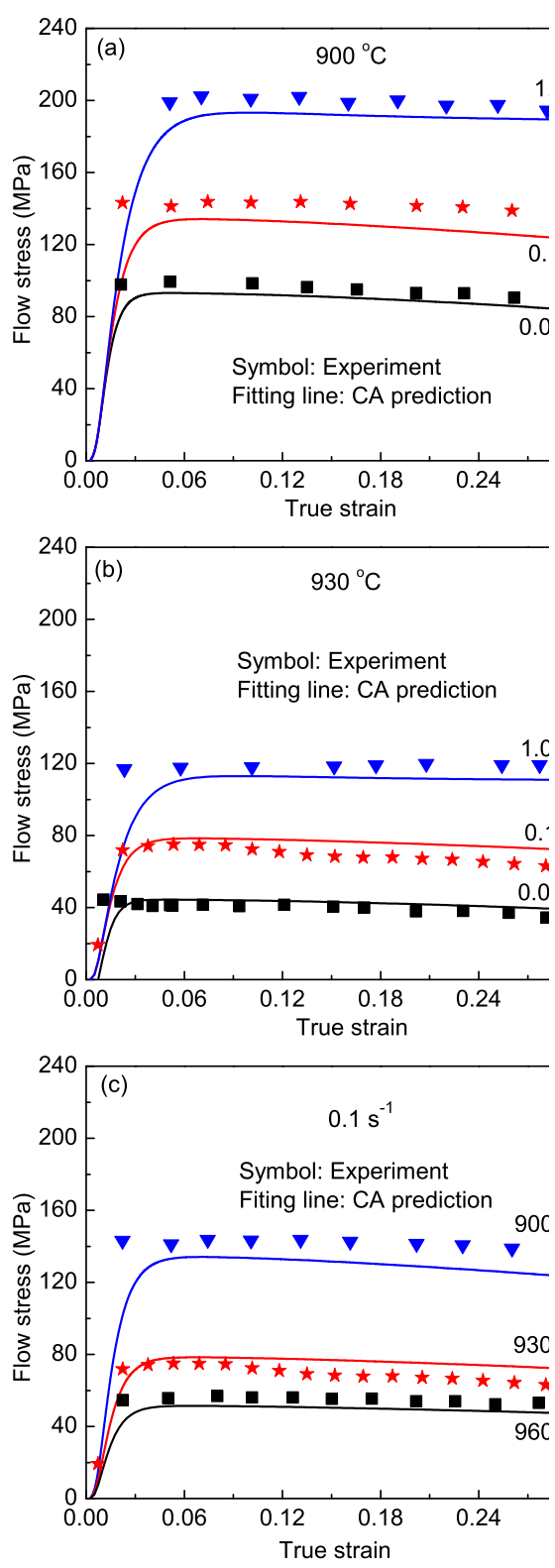

Fig. 13 Comparison of predictive flow stress with the experimental at different deformation conditions: (a) $900{ }^{\circ} \mathrm{C}, 0.01-1.0 \mathrm{~s}^{-1}$; (b) $930{ }^{\circ} \mathrm{C}$, $0.01-1.0 \mathrm{~s}^{-1}$; (c) $900-960{ }^{\circ} \mathrm{C}, 0.1 \mathrm{~s}^{-1}$

material is sensitive to deformation temperature and strain rate. Before the peak stress, the flow stress rises rapidly due to work hardening. However, the increasing rate of flow stress gradually slowed down due to the DRV. After the peak stress, an apparent flow softening can be observed under all deformation conditions. This phenomenon is mainly caused by the substructure evolution. During the transformation of the LABs to the HABs, both the dislocation reactions within the subgrains or the subgrain boundaries and the dislocation absorbed by the mobile subgrain boundaries could result in the reduction of dislocation density, which directly results in the reduction of flow stress. Furthermore, the extent of flow softening increases with the increasing strain rate or decreasing 
temperature, which corresponds well with the evolution tendency of dislocation density during subgrain evolution.

\section{Conclusions}

(1) The predicted results showed that the transformation of the LABs to the HABs was sensitive to strain rate, strain and temperature. The volume fraction of the HABs increased with the increasing strain rate or strain but decreased with temperature.

(2) The substructure evolution influenced the dislocation density. Both the dislocation reactions within the subgrains or the subgrain boundaries and the dislocation absorbed by the mobile subgrain boundaries could result in the reduction of dislocation density.

(3) The substructure evolution could result in the flow softening. The CA model considering the substructure evolution predicted the flow stress that agreed better with the experimental values.

\section{Acknowledgements}

This work was financially supported by the National Basic Research Program of China (No. 2010CB731701), the National Natural Science Foundation of China (Nos. 50935007 and 51175428), Foundation for Fundamental Research of Northwestern Polytechnical University in China (No. NPU-FFR-JC20100229), and the 111 Project (No. B08040).

\section{REFERENCES}

[1] Y.C. Zhu, W.D. Zeng and Y.Q. Zhao, Mater. Sci. Eng. A 552 (2012) 384.

[2] Z.C. Sun and H. Yang, Mater. Sci. Eng. A 523 (2009) 184.

[3] X.G. Fan, H. Yang, Z.C. Sun and D.W. Zhang, Mater. Sci. Eng. A 527 (2012) 5391.

[4] Z.C. Sun, H. Yang and G. J. Han, Mater. Sci. Eng.
A 527 (2010) 3464.

[5] D. He, J.C. Zhu, Z.H. Lai, Y. Liu and X.W. Yang, Mater. Design 46 (2013) 38.

[6] L.J. Huang, L. Geng and A.B. Li, Mater. Sci. Eng. A 505 (2009) 136.

[7] M.M. Tong, D.Z. Li and Y.Y. Li, Acta Mater. 52 (2004) 1155.

[8] H.G. Hesselbarth and I.R. Göbel, Acta Metall 39 (1991) 2135.

[9] R.L. Goetz and V. Seetharaman, Scr. Mater. 38 (1998) 405.

[10] D. Raabe, Acta Mater. 52 (2004) 2653.

[11] B.J. Yu, X.J. Guan, L.J. Wang, Q.K. Zeng, Q.Q. Liu and Y. Cao, Acta Metall. Sin. (Engl. Lett.) 24 (2011) 287.

[12] R. Ding and Z.X. Guo, Mater. Sci. Eng. A 365 (2004) 172 .

[13] F. Chen, Z.S. Cui, J. Liu, W. Chen and S.J. Chen, Mater. Sci. Eng. A 527 (2010) 5539.

[14] C.W. Zheng, N.M. Xiao, D.Z. Li and Y.Y. Li, Comput. Mater. Sci. 45 (2009) 568.

[15] Y. J. Lan, N.M. Xiao, D.Z. Li and Y.Y. Li, Acta Mater. 53 (2005) 991.

[16] F.J. Humphreys and M. Hatherly, Recrystallization and Related Annealing Phenomena, Pergamon Press, Britain, 2004, p. 202.

[17] R. Ding and Z.X. Guo, Acta Mater. 49 (2001) 3163.

[18] W.T. Read and W. Shockley, Phys. Rev. 78 (1950) 275.

[19] U.F. Kocks and H. Mecking, Prog. Mater. Sci. 48 (2003) 171.

[20] X.G. Fan and H. Yang, Int. J. Plast. 27 (2011) 1833

[21] S. Gourdet and F. Montheillet, Acta Mater. 51 (2003) 2685.

[22] F. Montheillet, O. Lurdos and G. Damamme, Acta Mater. 57 (2009) 1602.

[23] Y. P. Varshni, Phys. Rev. B 2 (1970) 3952.

[24] L. Jiao, M.Q. Li, X.L. Li and Y.P. Shi, Mech. Mater. 42 (2010) 157.

[25] J. Lin and J.B. Yang, Int. J. Plast. 15 (1999) 1181.

[26] J.S. Pan, J.M. Tong and M.B Tian, Foundation of Materials Science, Tsinghua University Press, Beijing, 2011, p. 227. (in Chinese) 